\title{
3D finite element simulations of high velocity projectile impact
}

\author{
Joško Ožbolt ${ }^{\mathrm{a}}$, Barış İrhan, and Daniela Ruta \\ Institute of Construction Materials, University of Stuttgart, 70569 Stuttgart, Germany
}

\begin{abstract}
An explicit three-dimensional (3D) finite element (FE) code is developed for the simulation of high velocity impact and fragmentation events. The rate sensitive microplane material model, which accounts for large deformations and rate effects, is used as a constitutive law. In the code large deformation frictional contact is treated by forward incremental Lagrange multiplier method. To handle highly distorted and damaged elements the approach based on the element deletion is employed. The code is then used in 3D FE simulations of high velocity projectile impact. The results of the numerical simulations are evaluated and compared with experimental results. It is shown that it realistically predicts failure mode and exit velocities for different geometries of plain concrete slab. Moreover, the importance of some relevant parameters, such as contact friction, rate sensitivity, bulk viscosity and deletion criteria are addressed.
\end{abstract}

\section{Introduction}

It is well known that in concrete structures the resistance, failure mode, crack pattern and crack velocity are strongly influenced by loading rate [1-13]. The rate dependent response of concrete is controlled through three different effects: (i) through the rate dependency of the growing micro-cracks (influence of inertia at the micro-crack level), (ii) through the viscous behaviour of the bulk material between the cracks (viscosity due to the water content) and (iii) through the influence of inertia of different kind, e.g. structural inertia, inertia due to the softening or hardening of the material or inertia related to the crack propagation. From the numerical point of view, assuming macro or meso scale analysis, the first two effects can be accounted for by the rate dependent constitutive law. Assuming that the resolution of underlying spatial discretization is fine enough, the third effect should be automatically accounted for through dynamic analysis where the constitutive law interacts with inertia [12].

Principally, with increase of loading rate failure mode tends to change from mode-I to mixed mode. As discussed in [12] and [13], responsible for this is inertia, which homogenizes the material of the impact zone and forces damage (crack) to move away from the zone of high inertia. Furthermore, when crack starts to propagate relatively fast, inertia at the crack tip tends to prevent crack propagation. Consequently, crack branching occurs and single crack splits into two inclined cracks. Moreover, significant inertia can be generated as a consequence of material softening and hardening. Elastic-brittle materials, such as glass, exhibit almost no influence of the cracking induced inertia on the rate dependent resistance. However, in quasi-brittle materials (e.g. concrete) and especially in ductile materials (e.g. steel) the inertia related to the propagation of macro-cracks significantly influence resistance and ductility of structures. For instance, it is well

\footnotetext{
${ }^{a}$ Corresponding author: ozbolt@iwb.uni-stuttgart.de
}

known that although steel exhibits almost no strain rate sensitivity, compared to quasi-static loading, its resistance increases with increasing loading rate and the response becomes more brittle. The reason for such behaviour is due to the fact that with increasing loading rate the size of the plastic zone at the crack tip reduces in size due to the action of inertia related to highly non-linear material response.

Recently, a 3D explicit finite element code has been developed for simulation of high velocity impact and fragmentation events [14]. In the code the rate sensitive thermo-mechanical microplane material model for concrete is used. In order to investigate the behaviour of concrete under high loading rates and verify the computational procedures developed, different problems were studied in the past, e.g. Compact Tension Specimen, Split Hopkinson Bar and Hammer Drop Tests [12], [15-17]. The comparisons between experimental and numerical results have shown that the code is able to correctly predict the phenomena related to high loading rate such as rate dependent resistance, crack branching, crack velocity and rate dependent failure mode. In the present study range of applicability of the code and validity of the underlying theories is tested by performing finite element simulations for some of the projectile perforation experiments documented in [18]. Note that exactly the same experiments have been studied numerically also by $[19,20]$.

\section{3D multi-body dynamic rate dependent $\mathrm{FE}$ analysis}

\subsection{Constitutive law-rate sensitive microplane model for concrete}

In the microplane model the material response is computed based on the monitoring of stresses and strains in different predefined directions. Integrating microplane stresses in a thermodynamically consistent way it is possible to

This is an Open Access article distributed under the terms of the Creative Commons Attribution License 4.0, which permits unrestricted use, distribution, and reproduction in any medium, provided the original work is properly cited. 
calculate macroscopic stress tensor. In the model, material is characterized by the uni-axial relations between stress and strain components on planes of various orientations. The microplane model used in this work is based on so-called relaxed kinematic constraint concept [21], which is a modification of the M2 microplane model proposed by Bažant and Prat [22]. More details on the model can be obtained from [21].

The rate dependency in the here used version of the microplane model for concrete accounts for two effects: (1) the rate dependency related to the formation (propagation) of the micro-cracks, which is the effect of inertia forces at the level of the micro-crack tip, and (2) the rate dependency due to the viscosity of concrete (bulk material) between the micro-cracks. The rate dependency for each microplane component is modeled based on the rate process theory [23-26]. It should be kept in mind that the rate sensitivity defines the influence of loading rate on the true material strength only, i.e. without any consideration of inertia at the macro scale. The inertia at the macro scale is automatically considered through dynamic analysis. The model parameters are calibrated based on the uniaxial compressive tests performed by [3], where the rate of applied loading was kept within the range so that inertia does not play a major role (less than $1 / \mathrm{s}$ ). The model was verified against experimental results for compressive and tensile behavior under different strain rates $[9,15,16]$. For example, in the case of compact tension specimen [16] it was confirmed that the progressive increase of resistance is due to the inertia generated at the tip of the macro crack, which causes crack branching and leads to progressive increase of resistance. Moreover, it was shown that the true tensile strength and fracture energy increase approximately linear in semi-log scale with increasing strain rate. The linear increase is controlled by the constitutive law (inertia at micro scale and influence of viscosity) whereas the progressive increase comes automatically from dynamic finite element analysis.

\subsection{Finite element formulation}

The dynamic nodal equilibrium equations are obtained by discretizing the weak (integral) form of balance equation for linear momentum in space. By constructing equilibrium equations at a known state and employing mass lumping, it is possible to update position of the nodes directly, without performing any iteration, using explicit time integration. In the presence of contact interactions, unfortunately, position update cannot be performed in single step because contact forces are not known beforehand. One can remedy such a situation by setting up a predictor-corrector type algorithm [14,27].

Artificial bulk viscosity should be applied to the numerical solution for two reasons: (i) to prevent elements from collapsing down due to high velocity gradients and (ii) to quiet truncation frequency "ringing" [28]. Bulk viscosity pressure is not a part of the constitutive response and can be used conveniently for all material models. It has been found in numerical simulations performed for concrete perforation experiments that the strengthening due to rate dependent micro-crack growth (material rate sensitivity) is not sufficient to produce necessary resistance against penetration. In addition bulk viscosity effects should also be present.

During projectile penetration motion function starts immediately losing its regularity around the contact region. In such a situation one should firstly consider if it is possible to heal motion function such that Lagrangian framework can be retained throughout the rest of deformation process. Among various possible alternatives, method based on adaptive element deletion has been employed for this purpose. In this technique underlying finite element discretization is kept in place. The problematic part of the body, where motion function started to lose its regularity, is simply removed from the system. Element deletion criteria play an important role and should be set with extreme care in order to avoid nonphysical solutions.

In all penetration simulations performed in this study, maximum principal strain has been used as a deletion criteria and its value has been set to 1.0. From the numerical results, it has been observed that stress state in some concrete elements around the contact region is almost hydrostatic compression. Such elements normally undergo very large deformations and still carry very high level of compressive stresses in the order of $1.0 \mathrm{GPa}$ when the maximum principal strain reaches the failure value. Since such elements can still transmit force and resist projectile, deletion of them might numerically reduce the resistance of concrete slab. Consequently, failure mode, exit velocity and all other relevant features might drastically change and simulation results turn out to be failure criteria value dependent.

Based on above discussion, deletion of elements with very high stress is acceptable if and only if it does not change the results of numerical simulation significantly. Elements with very high stress can be conveniently deleted for penetration simulations if the following two conditions are satisfied. (i) Contact algorithm should not be influenced from the deletion of elements with very high stress too much. As a concrete element gets into contact with projectile, it deforms and also its kinetic energy is increased. If this element is continuously in contact, after a while nodes of this element, which contribute to contact, start to move together with projectile and, therefore, contact forces transmitted over such an element gradually lose their intensity. Only after then deletion of such an element will not disturb contact behavior and therefore is allowed even if it still carries very high stress. (ii) Response of concrete slab should not be altered too much as the concrete elements with very high stress are deleted. This is only possible if there is an internal mechanism which brings back approximately the nodal forces removed due to element deletion. This effect, of course, should be produced by the undeleted elements connected to those nodes. The nodal internal forces in those elements should be adjusted such that dynamic equilibrium prior to element deletion is nearly recovered. Such an artificial strengthening is possible due to existence of material rate dependency and bulk viscosity pressure. However such a recovery has not been observed at projectile penetration simulations because at the onset of element deletion strain rates are already very high and as a consequence artificial strengthening is negligibly small. 
(a)
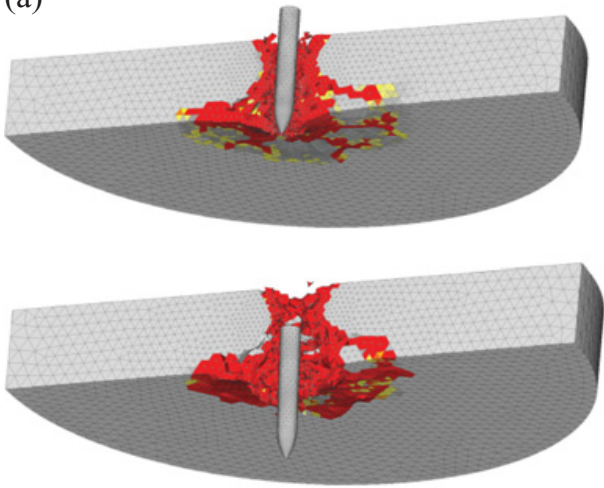

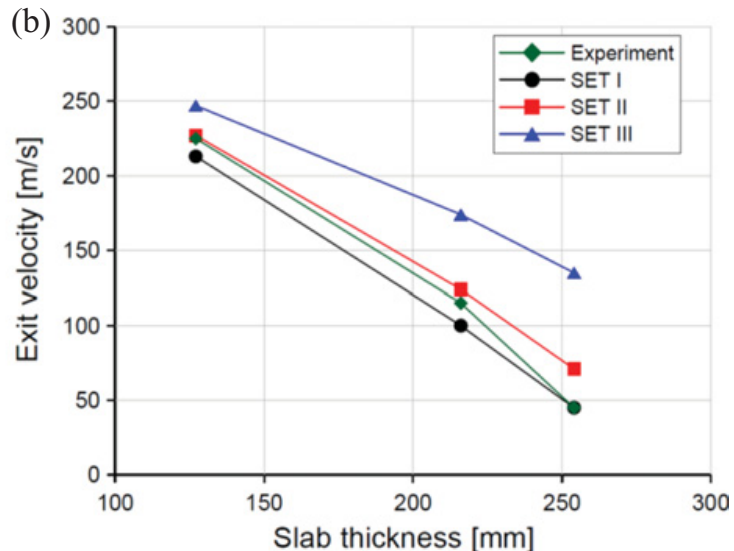

Figure 1. (a) 3D finite element discretization with two typical sequences of penetration events for SET-I/CS216 (section cut); (b) Relation between exit velocity and slab thickness for all three sets of analyses.
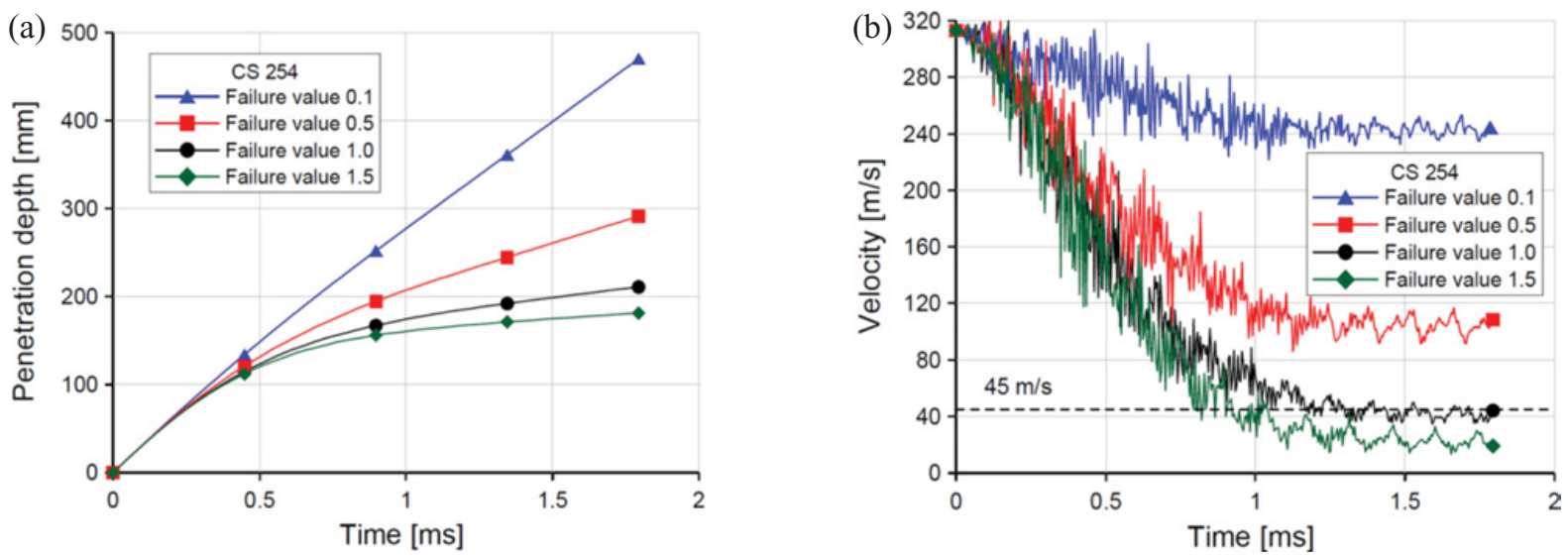

Figure 2. Time history graphs for different failure values: (a) Penetration depth vs. time and (b) Projectile velocity vs. time.

\section{Projectile perforation of concrete slab}

\subsection{Experimental investigations}

To test the performance of the developed 3D explicit finite element research code at very high loading rates series of numerical simulations have been performed for some of the projectile perforation experiments documented in [18]. In the experiments plain concrete circular slabs of different thickness were tested against penetration by using ogivenose shaped projectiles with an initial impact velocity of approximately $313 \mathrm{~m} / \mathrm{s}$. Further details about experiments can be found in [18].

\subsection{Numerical investigations}

The numerical simulations have been carried out for three slab thicknesses $(t=127,216$ and $254 \mathrm{~mm}$, Fig. 1a) with entire geometry, i.e. no symmetry was utilized. The objectivity of the results with respect to the mesh size is assured by the use of the crack band method [29]. In the analysis maximum principal strain has been used as failure criteria for the deletion of elements.

Failure value was set as equal to 1.0. To simplify notation we will denote $127 \mathrm{~mm}$ circular slab as CS127 and so on. Rate dependent microplane material model [21] has been used for concrete.

For projectile Saint-Venant Kirchhoff hyper-elastic material model is adopted assuming that projectile would undergo little or no deformation during perforation. First, a parametric study has been conducted in order to investigate the significance of concrete rate sensitivity and friction between circular slab and projectile on the results. For each slab thickness three sets of simulations have been performed: (I) rate-on/friction-on, (II) rate-on/friction-off and (III) rate-off/friction-off. Friction coefficient is set to 0.5 .

The typical failure mode SET-I/CS216 that is in very good agreement with experimental observation is shown in Fig. 1a. As can be seen from Fig. 1b, for SET-I (rateon/friction-on) exit velocities are in very good agreement with experiments for all slab thicknesses. The results for SET-II (rate-on/friction-off) also exhibit relatively good agreement with test results. It can be seen that friction does not significantly influence the exit velocity. On the other hand, results obtained from SET-III (rate-off/friction-off) are not objective. The exit velocity becomes very sensitive to rate effect as the thickness of the slab increases. This suggests that in case of high velocity impact problems not only inertia but also rate sensitivity influences the 

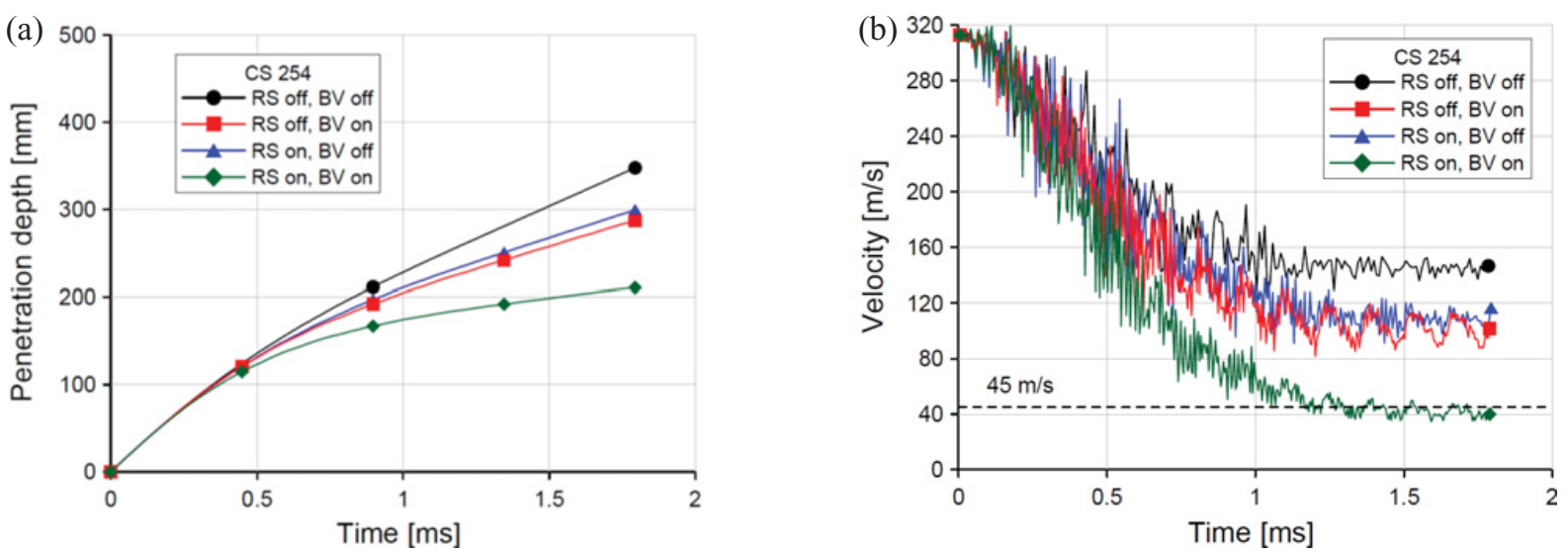

Figure 3. Influence of rate sensitivity and bulk viscosity on time history response: (a) Penetration depth vs. time and (b) Projectile velocity vs. time.

response. It is reasonable that with increase of structural size (thickness of the slab) rate sensitivity becomes more important. Namely, due to the large volume of activated material the structural resistance increases.

As has been discussed in previous section, it is very difficult to predict an optimum failure value beforehand for deletion criterion based on maximum principal strain, especially from contact algorithm point of view, if there are some elements with very high stress at the onset of element deletion. For this reason, set of numerical simulations have been performed to study the sensitivity of numerical results with respect to failure value. Simulations have been performed for failure values (maximum principal strain) $0.1,0.5,1.0$ and 1.5 using SET-I/CS254.

As can be seen from Fig. 2, numerical simulations are very sensitive to the failure value especially for smaller ones. On the other hand, there is a convergence of results as the failure value increases. Based on these observations, it can be concluded that optimum value should be somewhere between 1.0 and 1.5.

To avoid numerical stability problems and maintain the robustness of underlying contact algorithm, failure value has been set as equal to 1.0 in all the simulations. It should be noted that even if the failure value is set to 1.5 , no significant change in the overall response is observed.

To investigate the importance of material rate sensitivity (RS) and bulk viscosity (BV) three additional simulations, first one with RS-on/BV-off, the second one with RS-off/BV-on and the third one with RS-off/BV-off have been performed using SET-I/CS254.

The important results of these simulations are shown in Fig. 3 together with those for the case RS-on/BV-on. As can be seen from Fig. 3, the resistance of the slab is reduced dramatically if both contributions are set to off. Moreover, the resistance is reduced almost for the same amount when one parameter, either RS or BV, is set to off. Based on this observation it can be concluded that both RS and BV are very important and should be included in the numerical simulations for correct prediction of concrete resistance against penetration. Moreover, this suggests that the volumetric strain rate of concrete in front of the projectile tip significantly contributes to the resistance against penetration.

\section{Conclusions}

In the present article recently developed 3D explicit finite element code for simulation of high velocity impact and fragmentation events is briefly discussed. The code is then used in the simulation of the projectile perforation of the plain concrete slab. Based on the results of the numerical simulations the following conclusions can be drawn out. (1) The comparison between experimental and numerical results shows that the employed $3 \mathrm{D}$ FE code based on the rate sensitive microplane model for concrete is able to realistically predict exit velocities and crater profiles for all three slab thicknesses. (2) Based on the results of the parametric study it can be concluded that in the case of high velocity, impact rate sensitivity of concrete cannot be neglected. It significantly contributes to the resistance, especially if the size (slab thickness) of concrete specimen is large. (3) In contrast to rate sensitivity, friction between projectile and concrete does not significantly contribute to the resistance. (4) The choice of deletion criteria of finite elements significantly influences the results of the analysis. It is shown that maximum principal strain of 1.0 is a reasonably good choice for the failure value of concrete elements. It is shown that failure values larger than 1.0 do not significantly influence exit velocities and failure mode. (5) It is found that both material rate sensitivity and bulk viscosity influence the response. For the investigated case the influence of bulk viscosity of concrete is approximately the same as the influence of concrete rate sensitivity. For realistic analysis both contributions should be accounted for. This suggests that the volumetric strain rate of concrete in front of the projectile tip significantly contributes to the resistance against penetration.

\section{References}

[1] L. Freund, Crack propagation in an elastic solid subjected to general loading - I. Constant rate of extension, Journal of the Mechanics and Physics of Solids, 20(3), 129-140 (1972)

[2] L. Freund, Crack propagation in an elastic solid subjected to general loading - II. Non-uniform rate of extension, Journal of the Mechanics and Physics of Solids, 20(3), 141-152 (1972) 
[3] W. Dilger, R. Koch, and R. Kowalczyk, Ductility of plain and confined concrete under different strain rates, ACI Journal Proceedings, 81(1), 73-81 (1984)

[4] N. Banthia, S. Mindess, and A. Bentur, Impact behaviour of concrete beams, Materials and Structures, 20(4), 293-302 (1987)

[5] H.-W. Reinhardt, Concrete under Impact Loading, Tensile Strength and Bond, HERON, 27(3) (1982)

[6] P. Bischoff and S. Perry, Compressive behaviour of concrete at high strain rates, Materials and Structures, 24(6), 425-450 (1991)

[7] J. Weerheijm, Concrete under impact tensile loading and lateral compression, TU Delft, the Netherlands (1992)

[8] J. Ožbolt and H.-W. Reinhardt, Rate dependent fracture of notched plain concrete beams, CONCREEP 7, 57-62 (2005)

[9] J. Ožbolt, K. K. Rah, and D. Meštrović, Influence of loading rate on concrete cone failure, International Journal of Fracture, 139(2), 239-252 (2006)

[10] M. Larcher, Development of discrete cracks in concrete loaded by shock waves, International Journal of Impact Engineering, 36(5), 700-710 (2009)

[11] R. R. Pedersen, Computational modelling of dynamic failure of cementitious materials, Delft University of Technology (2010)

[12] J. Ožbolt, A. Sharma, and H.-W. Reinhardt, Dynamic fracture of concrete-compact tension specimen, International Journal of Solids and Structures, 48(10), 1534-1543 (2011)

[13] J. Ožbolt and A. Sharma, Numerical simulation of dynamic fracture of concrete through uniaxial tension and L-specimen, Engineering Fracture Mechanics, 85, 88-102 (2012)

[14] B. İrhan, High velocity impact and fragmentation of concrete: Numerical simulation, Institut für Werkstoffe im Bauwesen der Universität Stuttgart (2014)

[15] J. Ožbolt, A. Sharma, B. İrhan, and E. Sola, Tensile Behavior of Concrete under High Loading Rates, International Journal of Impact Engineering, 69, 55-68 (2014)

[16] J. Ožbolt, J. Bošnjak, and E. Sola, Dynamic Fracture of Concrete Compact Tension Specimen: Experimental and Numerical Study, International Journal of Solids and Structures, 50, 4270-4278, (2013)
[17] N. Bede, J. Ožbolt, A. Sharma, and B. İhan, Dynamic fracture of notched plain concrete beams: 3D finite element analysis, International Journal of Impact Engineering, 77, 176-188 (2015)

[18] J. D. Cargile, Development of a constitutive model for numerical simulation of projectile penetration into brittle geomaterials, Technical Report SL-99-11, U.S. Army Engineer Research and Development Center, Vicksburg, MS (1999)

[19] F. C. Caner and Z. P. Bažant, Impact comminution of solids due to local kinetic energy of high shear strain rate: II-Microplane model and verification, Journal of the Mechanics and Physics of Solids, 64, 236-248 (2014)

[20] A. O. Frank, M. D. Adley, K. T. Danielson, and $\mathrm{H}$. S. McDevitt Jr, The high-rate brittle microplane concrete model: Part II: application to projectile perforation of concrete slabs, Computers and Concrete, 9(4), 311-325 (2012)

[21] J. Ožbolt, Y. Li, and I. Kožar, Microplane model for concrete with relaxed kinematic constraint, International Journal of Solids and Structures, 38(16), 2683-2711 (2001)

[22] Z. P. Bažant and P. C. Prat, Microplane model for brittle-plastic material: I. Theory, Journal of Engineering Mechanics, 114(10), 1672-1688 (1988)

[23] H. Mihashi and F. Wittmann, Stochastic Approach to Study the Influence of Rate of Loading on Strength of Concrete, HERON, 25(3) (1980)

[24] A. S. Krausz and K. Krausz, Fracture kinetics of crack growth, Springer, 1 (1988)

[25] Z. P. Bažant et al., Large-strain generalization of microplane model for concrete and application, Journal of Engineering Mechanics, 126(9), 971-980 (2000)

[26] Z. P. Bažant, F. C. Caner, M. D. Adley, and S. A. Akers, Fracturing rate effect and creep in microplane model for dynamics, Journal of Engineering Mechanics, 126(9), 962-970 (2000)

[27] N. J. Carpenter, R. L. Taylor, and M. G. Katona, Lagrange constraints for transient finite element surface contact, International Journal for Numerical Methods in Engineering, 32(1), 103-128 (1991)

[28] L. M. Taylor and D. P. Flanagan, PRONTO 3D: A three-dimensional transient solid dynamics program (1989)

[29] Z. P. Bažant and B. Oh, Crack band theory for fracture of concrete, Materials and Structures: 155-177 (1983) 